\title{
Energy Aware - Bio-Inspired Hybrid WSN for Area Surveillance (E-BHAS)
}

\author{
Mininath Nighot, Ashok Ghatol and Vilas Thakare \\ Sant Gadge Baba Amravati University, Amravati - 444602, Maharashtra, India; \\ imaheshnighot2gmail.com, vc_2005@rediffmail.com, vilthakare@yahoo.co.in
}

\begin{abstract}
Objectives: Randomly moving target search in an unknown location is a complex problem which does not have a linear solution. This paper proposes Energy aware - Bio-inspired Hybrid technique for target finding Area surveillance application. Methods/Statistical Analysis: In recent years, many researchers tried to find a generic solution which requires minimum energy for target searching problem. For the proposed work four algorithms are proposed which are MSN's Movement Prediction Algorithm (MMPA), Leader Selection Algorithm (LSA), and Leader's Movement Predication Algorithm (LMPA), and Follower's Algorithm. Particle Swarm Optimization (PSO) technique is used to move the Mobile Sensor Nodes (MSN) in searching area. Findings: There is a need to study the hybrid or heterogeneous Sensor network (combination of the Static sensor network and Mobile sensor network) as it is less studied in research literature. The major hurdle is communication between heterogeneous sensor nodes. Due to the mobility of MSN, topology changes frequently and it affects the communication in the network. In area surveillance domain, finding the randomly moving target using sensor node is a complex and non-linear problem. There is no straight forward solution. Nature based techniques may be more effective for such problem. Bio-inspired Hybrid WSN technique is to be studied for its suitability for target finding and target tracking application in area surveillance domain. To perform the work, two scenarios are considered and calculated their energy to prove that ifmore number of SSNs is planted, the target can be found in less time and ultimately overall energy of network reduces. Through the simulation, it is shown that E-BHAS reduces the energy when more number of SSNs is involved in the network. Application/ Improvements: Borderline Surveillance, Disaster Management, under water Surveillance, Traffic Monitoring etc.
\end{abstract}

Keywords: Area Surveillance, Energy, Hybrid Wireless Sensor Network, Particle Swarm Optimization

\section{Introduction}

Wireless Sensor Networks are mainly studied by researchers due to its various applications such as area surveillance, health care monitoring environment monitoring. Sensors have their own physical properties like temperature, moisture, smoke, light, door, etc. As per the demands of the application, specific sensors are to be used. The main challenges in WSN are its Processing power, limited memory, and low bandwidth. Most of the energy is drained for the communication between sensor nodes $\frac{1,2}{}$. If the communication overhead is reduced in the network, then the overall time of the network will increase. Researchers need to consider these limitations of WSN to provide a solution. In recent work, very few researchers focused on hybrid WSN due to communication hurdle between SSN to MSN.

To reduce the intercommunication of nodes, distributed approach is most suitable. In distributed approach,

*Author for correspondence 
each node takes their decisions and processes it independently. Bio-inspired techniques such as ant colonies, swarms of the particle, fish schooling, etc. work in a distributed manner. The techniques can be used to increase the lifetime of the network. The number of computations depends on the size of the problem. If problem size increases, then a computation also increases exponentially. It is more efficient for the increased problem size or when the problem is complex ${ }^{3,4}$.

The objectives of the paper are: 1 . to simulate random moving target searching in unknown environment, with minimum sensor nodes in hybrid WSN (SSN and MSN) to reduce energy, and 2. to efficiently use of PSO (Particle Swarm Optimization) technique to achieve group movements of MSNs for target searching with minimum energy, which is explained below.

\subsection{PSO- Particle Swarm Optimization}

Self-organization is a nonlinear distributed system which cannot have a linear solution and is not controlled by any single particle. It is a continuous process in which particles interact with each other locally.

Initially,self-organized systems are predictable, but after some iteration or some time instances, these may be predictable, neutral or unpredictable.

There are mainly five features:

1. Positive feedback,

2. Negative feedback,

3. Amplification,

4. Multiple iterations, and

5. Balance of exploitation and exploration.

The system has positive and negative feedback in which positive feedback inspires for the creation of convenient structure while negative feedback balances the positive feedback $\frac{5-7}{}$.

\subsection{PSO Algorithm}

Generally, PSO performs searching operations using a swarm of particles. To get the optimal position each particle move in the direction to their best local position (pbest) and best global position (gbest) ${ }^{8-10}$.

$$
\operatorname{pbest}(i,(k-1))=\underset{k=1,2 \ldots t}{\arg \min }\left[f\left(P_{i}(k)\right)\right],
$$

Where, $f(x 1, x 2, \ldots x n)$

$$
\begin{gathered}
\operatorname{gbest}(k-1)=\underset{i=1,2 \ldots N}{\arg \min }\left[f\left(P_{i}(k)\right)\right] \\
k=1,2 \ldots \mathrm{t}
\end{gathered}
$$

Where, $\mathrm{t}$ is number of iterations and $\mathrm{N}$ is the total number of particles (i.e. swarm size).

Each particle in the swarm decides the movement by an objective function:

$$
f(x 1, x 2, \ldots x n)
$$

where, $f: \Re^{n} \rightarrow \Re$.

The fitness of particle is calculated from its pbest position in searching the area. The particle where pbest is closer to the gbest have lower cost and vice versa. PSO determines minimization of a fitness function. After each iteration, the position of a particle and its velocity are modified to achieve lower cost or higher fitness value. The notations used in PSO are shown in Table 1.

Velocity and position of every particle are modified after $\mathrm{k}$ iteration and is shown as:

$$
\begin{aligned}
& V_{i}^{d}(k)=\omega V_{i}^{d}(k-1)+c 1 \text { rand } 1_{i}^{d}(k) \\
& \quad\left(\text { pbest }_{i}^{d}-X_{i}^{d}\right)+c 2 \operatorname{rand} 2_{i}^{d}(k) \\
& \quad\left(\text { gbest }^{d}-X_{i}^{d}\right)
\end{aligned}
$$

$$
X_{i}^{d}(k)=X_{i}^{d}(k-1)+V_{i}^{d}(k)
$$

Here rand $1 \&$ rand 2 are random numbers in the range for good coverage. $\omega$ is: $0.2 \prec \omega \prec 1.2$ an inertia 
Table 1. The notations used in PSO

\begin{tabular}{|c|c|}
\hline Symbol & Meaning \\
\hline$c 1$ & Self confidence factor \\
\hline$c 2$ & Swarm confidence factor \\
\hline rand $1 \&$ rand 2 & Random numbers \\
\hline$\omega$ & Inertia weight \\
\hline$x_{i}^{d}$ & Particle's position \\
\hline$V_{i}^{d}$ & Particle's velocity \\
\hline$k, k-1$ & $\begin{array}{l}\text { Current and previous iterations } \\
\text { respectively (movement of } \\
\text { particle) }\end{array}$ \\
\hline pbest $_{i}^{d}$ & Particles best position \\
\hline gbest $^{d}$ & Swarm's best position \\
\hline$t$ & Total number of iterations \\
\hline$d$ & Dimensions of solution space \\
\hline$P_{i}$ & $\begin{array}{c}\text { Position of agents in the solution } \\
\text { space }\end{array}$ \\
\hline
\end{tabular}

weight manipulates the trade off between exploitation and exploration abilities of the object.

The rest of the paper is organized as follows: A literature survey is explained in Section 2. In Section 3, proposed work with algorithms are introduced and explained in detail. Results analysis is given in Section 4 and 5 conclusions and future scope is given.

\section{Literature Survey}

In recent years, several researchers have proposed different techniques to find the target, but most of them are based on SSN and very few are based on MSN and hybrid (MSN and SSN). Bio-inspired optimization strategies are also implemented to optimize the performance of their work. Due to the limitations in sensing, processing, and energy of sensor node, it becomes difficult to find the target if the target is moving randomly. Some of the techniques are now discussed.

Target searching by MSN is more complex when the target moves randomly. Due to its unpredictable movement, it is very difficult to predict its exact position. To track the target, an effective navigation technique is required. Kalman filter is good technique ${ }^{11}$, which uses geometric predictive location tracking algorithm. $\mathrm{In}^{12}$ proposed extended kalman filter in Time Of Arrival (TOA) measurement model for searching the target. For better tracking, distributed MSNs are used $\frac{13-15}{15}$ implemented distributed target tracking system which performs better in energy consumption and loss of connectivity. $\operatorname{In}^{16}$ implemented a solution on dynamic allocation of nodes to trace the multiple targets. Each sensor node can operate in two modes either data collection mode or tracking mode. Distributed sensor allocation algorithm is implemented to find the dynamic allocation $\frac{17,18}{}$ proposed a couple of algorithms to track the target in the totally unknown physical environment. Multiple robots search the target cooperatively to get the parameter ranges of cooperation method in multiple robots using a combination of HRL and MAXQ algorithms. All parameters which are required for cooperation are obtained through learning approach and the new task can be performed by the multiple robots. With simulation study, it is studied and shown that multiple robots in the unknown environment can search the target ${ }^{19}$ investigates a new technique to navigate mobile sensor node to track a mobile target in an unknown environment. Min-Max approximation 
algorithm is used to track the target and cubic function for sensor navigation. The weighted tracking algorithm is implemented to exploit the measurement information. Results are compared with Time Difference of Arrival (TDOS) with Kalman filter. Coverage quality of connectivity of the network can be enhanced by deploying MSNs in the network ${ }^{20-23}$ proposed a hybrid network which consists of static and mobile sensors. Author proposed minimum number of MSNs and SSNs are divided in a large number of group and how SSN and MSN communicate.

$\mathrm{In}^{24}$, proposed a solution on energy efficient coverage and its energy consumption. Stationary nodes and mobile nodes are used for simulation. Grid exclusion algorithm and Dijkstra's algorithm are used for nodes coverage rate. To optimize communication energy consumption, Hybrid algorithm Distributed Particle Swarm optimization and Simulated Annealing (DPSOSA) is implemented. Free space propagation model is used to calculate energy consumption. Results are compared with PSO and Shown that DPSOSA optimizes coverage and communication energy ${ }^{25,26}$, focused problem of tracking and monitoring a mobile target in the field with multiple obstacles for increasing network lifetime. MSN's are located using relocation technique. The proposed work finds nearoptimal relocation strategy for MSN. It also finds energy efficient path to send information from movable target to destination. T map the field into grid diagraphs are used. Proposed technique is proved with simulation result and shown network lifetime increases.

\section{Proposed Work}

Proposed technique shows self-organized MSN and SSN to track the moving target (T) using PSO. MSN and SSN are equipped with Global Positioning System (GPS) which is used to share their exact location coordinates in two-dimensional spaces. Target is assumed to move randomly. Without having visual sense and directional guidance to MSN and SSN, using only location guidance, MSN tracks the moving target.

The proposed system is heterogeneous, using both SSN and MSN in the same system. SSNs have the capability to send their own locations to all MSNs which are far away. If the target is in the Searching Range (SR) of SSN then SSN sends its own location to all MSN.

MSN are low capability sensors having a movable trolley/vehicle on which MSN's are located. The trolley moves as per the signals are given by MSN. It can move in only three directions Left (Lt), Straight (St) and Right (Rt), from the current position. Left and right rotations are exactly 45 degrees from the current position. Figure 1 shows the possible rotations from the current position. There are 8 possible initial positions of MSN and each

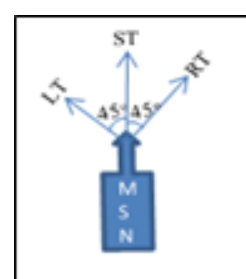

(a)

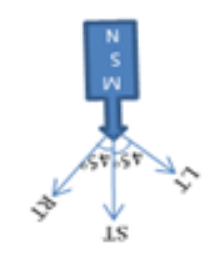

(e)

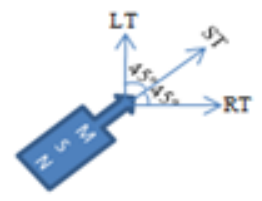

(b)

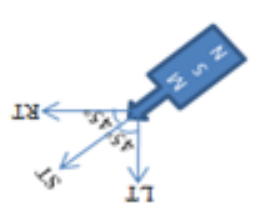

(f)

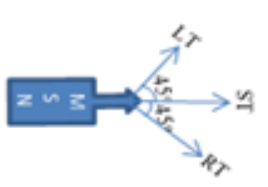

(c)

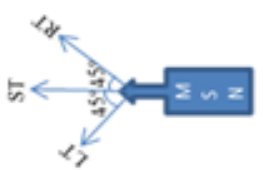

(g)

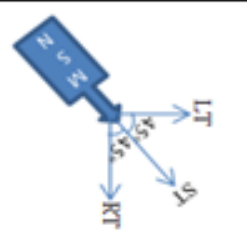

(d)

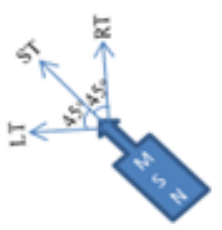

(h)

Figure 1. The possible rotations from current position. 
position has its relative left, straight and right rotations for movement.

SR is a range or radius of MSN and SSN in which it can search the target. If the target comes in the SR of any MSN, it detects the target. Communication Range (CR) / radio range is a radius of MSN in which MSNs can communicate with each other for sharing their location coordinates and sharing a message mentioning the target is in its SR.

\subsection{Topology Formation by MSN}

All MSNs together forms a swarm. MSN moves randomly to search the target, but it must be in the range of at least one MSN's CR. Initially, no two or more MSNs will be in others SR. Any two MSNs are a 2SR distance away from each other. If two MSNs are sharing SR, then same space will be searched by these two MSNs. By using this initial condition, the search space is increased. Figure 2 shows the possible minimum and maximum distance between two MSN. Figure 2(a) shows MSNs are sharing other's SR. In such case, same area will be searched by multiple MSNs and is wastage of time. Figure 2(b) shows the minimum distance between two MSN i.e. 2SR. So that it will search in the unsearched area. Figure 2(c) shows the maximum distance between two MSN i.e. CR to communicate with each other. If distance is increased than CR then sensors will not be able to communicate with each other.

\subsection{The Basic Flow of Systems is as Follows}

Initially, SSNs are placed randomly in the searching space. Target $(\mathrm{T})$ is also placed randomly and is movable. It moves randomly and is not GPS equipped. Swarm of MSNs are placed at any random place or at the border of searching space. MSNs move randomly by swarm technique. While moving randomly if $\mathrm{T}$ found, then the mission is complete, else searching process continues.

Due to the movement of $\mathrm{T}$, if it comes in the SR of any SSN then SSN broadcasts its own location coordinates and a message saying 'Target is found' to all MSNs. Once location and message received by all MSNs, all MSNs calculate its own distance from sender SSN using Euclidean distance.

$$
d_{i}=\sqrt{\left(x-x_{i}\right)^{2}+\left(y-y_{i}\right)^{2}}
$$

Where $(x, y)$ coordinates of SSN and $\left(x_{i}, y_{i}\right)$ coordinates of particles.

All MSNs share their calculated distance from Sender SSN (SSSN) to each other. The MSN which is closer to SSSN will be selected as a Leader and others will be followers. Leader MSN (LMSN) will decide its direction to reach to SSSN and move step by step towards SSN. Other MSN will not waste their energy for deciding the direction and path. They will just follow the LMSN. At every

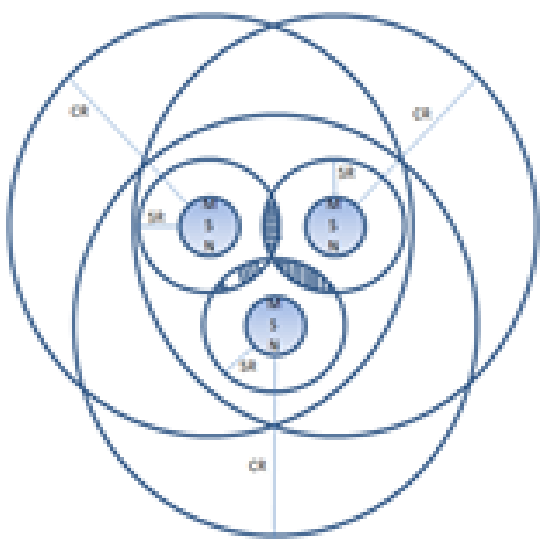

(a)

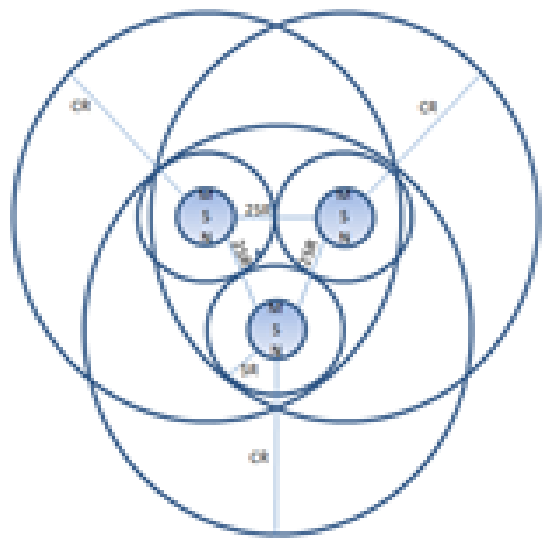

(b)

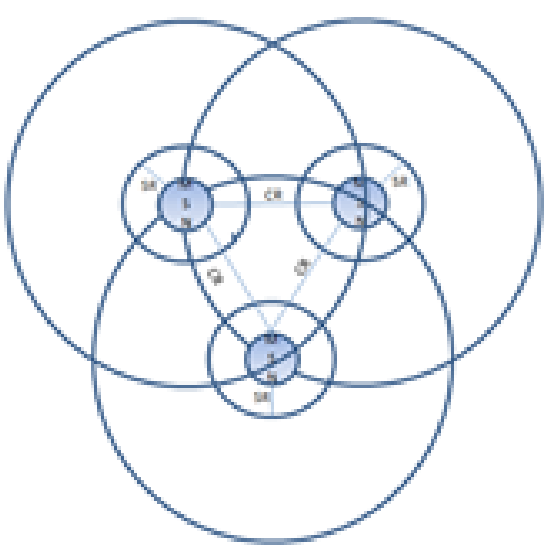

(c)

Figure 2. (a) Shows that the dashed area is searched by both sensors which are not required, (b) Shows at least 2SR should be the distance between MSN, and (c) shows worst case scenario of sensors (maximum distance between two sensors). 
step, all MSNs calculate their current distance from SSSN and share it to all MSNs. If any Follower MSN (FMSN) is closer to SSSN than LMSN, it will be selected as a new LMSN and remaining will be FMSN. In such fashion, MSNs travels to SSSN, without knowing directions and any manual interaction. Once all MSNs reach to SSN, they start searching again with their random search.

\subsection{Energy Module}

As mentioned in $\mathrm{LEACH}^{27}$ First order Radio Model is used. Radio dissipates $50 \mathrm{~nJ} / \mathrm{bit}$ for transmitting and receiving and for an amplifier $100 \mathrm{pJ} / \mathrm{bit} / \mathrm{m}^{2}$, the energy loss due to channel transmission, so for ' $k$ ' bit message and ' $d$ ' distance transmitting and receiving energy can be calculated as:

Transmitting Energy,

$$
\begin{aligned}
& E_{T X}(k, d)=E_{T X-e l e c}(k)+E_{T X-\text { amp }}(k, d) \\
& E_{T X}(k, d)=E_{\text {elec }} * k+\varepsilon_{\text {amp }} * k * d^{2}
\end{aligned}
$$

Receiving Energy,

$$
\begin{aligned}
& E_{R X}(k)=E_{R X-\text { elec }}(k) \\
& E_{R X}(k)=E_{\text {elec }}(k)
\end{aligned}
$$

\subsection{Proposed Algorithms}

To search the target, four algorithms are proposed which are as follows:

1. MSN's Movement Prediction Algorithm (MMPA) for searching (T),

2. Leader Selection Algorithm (LSA),

3. Leader's Movement Predication Algorithm (LMPA) for traveling towards SSSN, and

4. Follower's Algorithm.

\section{Algorithms:}

\subsubsection{MSN's Movement Prediction Algorithm (MMPA) for Searching (T)}

The flowchart shown in Figure 3 explains the next movement prediction of MSN while searching the target.

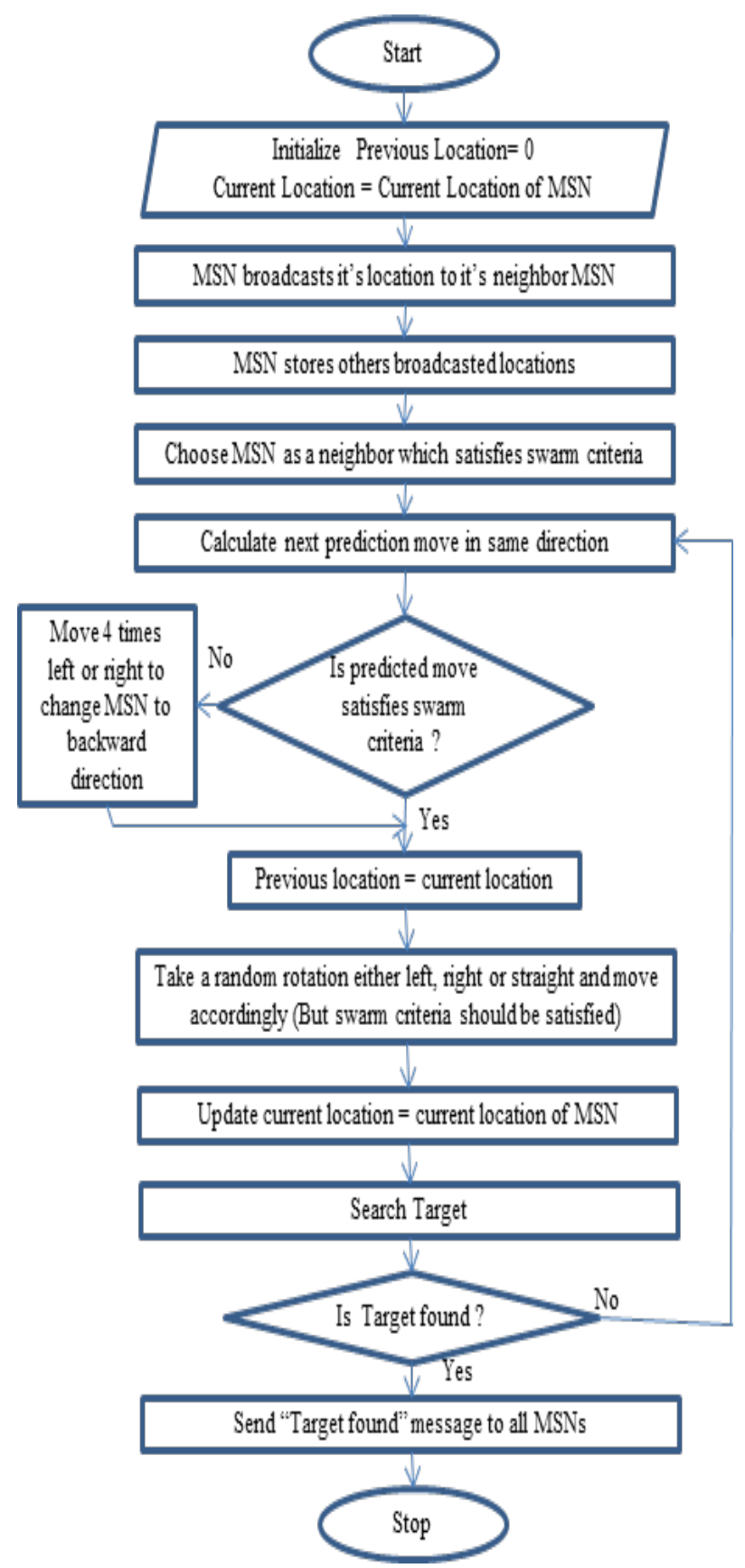

Figure 3. Flowchart of MMPA. 


\subsubsection{Leader Selection Algorithm (LSA)}

While moving towards to the message sender SSN, if any MSN is nearer to the SSN, then it will be selected as a leader as explained in the flowchart it is shown in Figure 4.
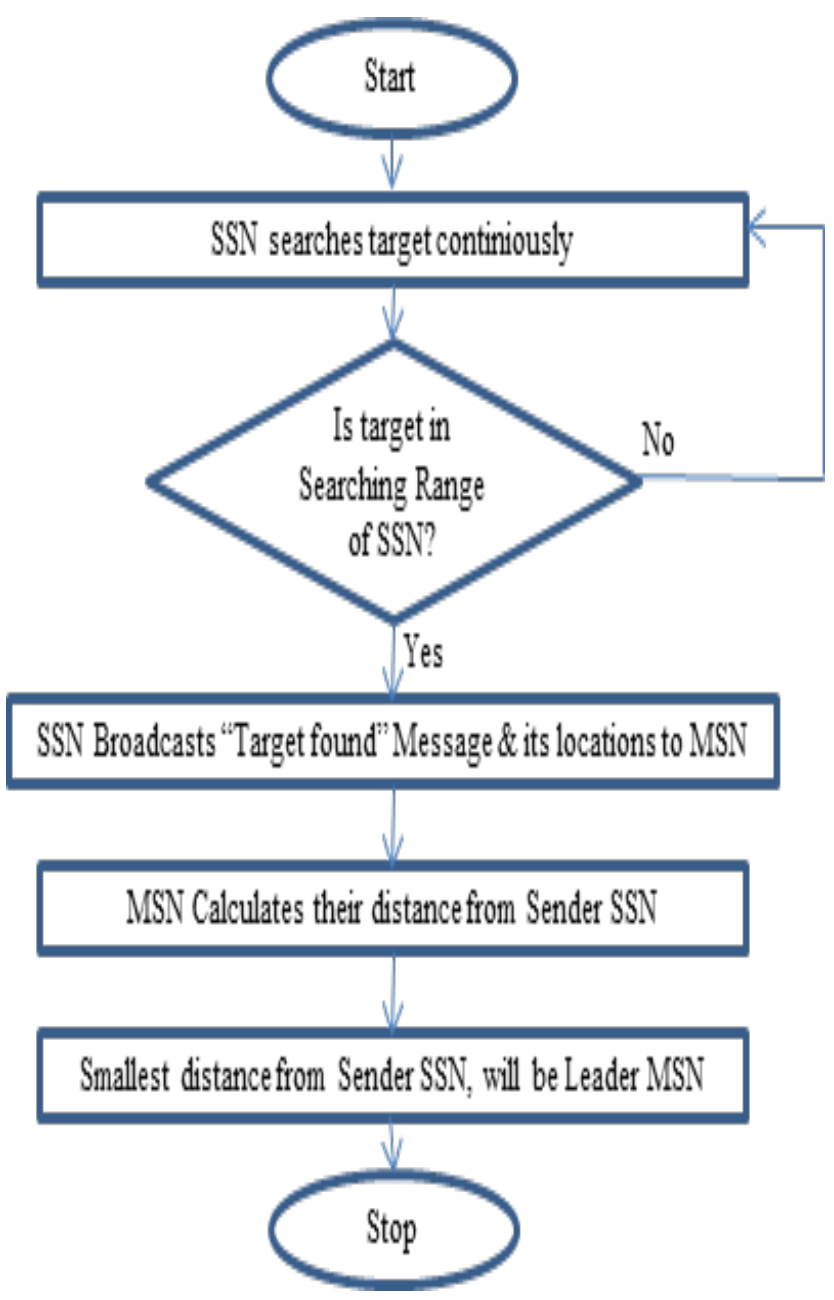

Figure 4. Flowchart of LSA.

\subsubsection{Leader's Movement Predication Algorithm (LMPA) for Traveling Towards SSSN}

Once the leader is selected, the leaderneeds to travel towards sender SSN using most suitable shortest path. Prediction of movement is explained in Figure 5 using a flowchart.
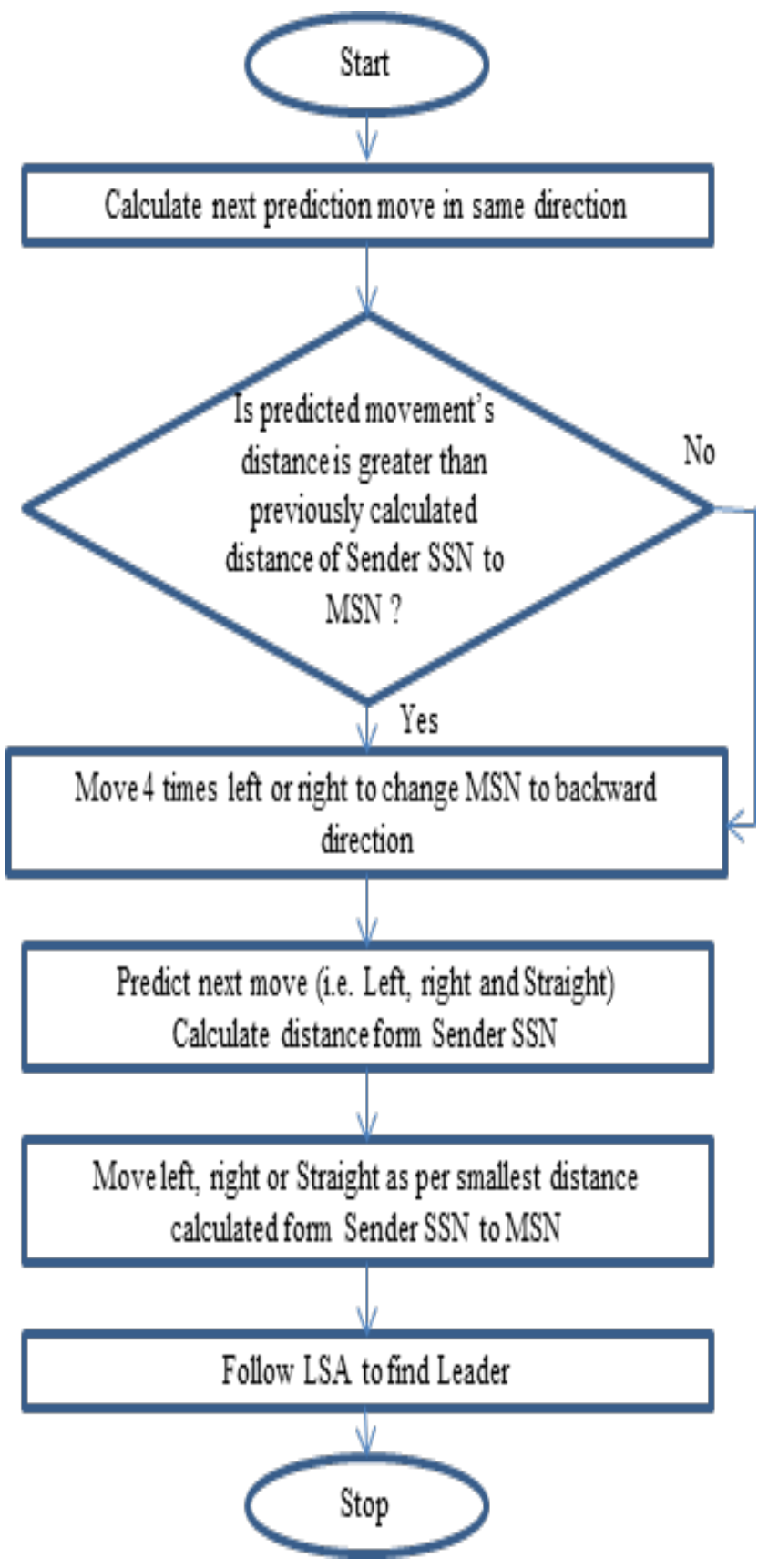

Figure 5. Flowchart of LMPA.

\subsubsection{Follower's Algorithm}

As per the movement and directions of leader MSN, other MSNs need to follow its path. So it acts like a follower of leader and is explained in Figure 6. 


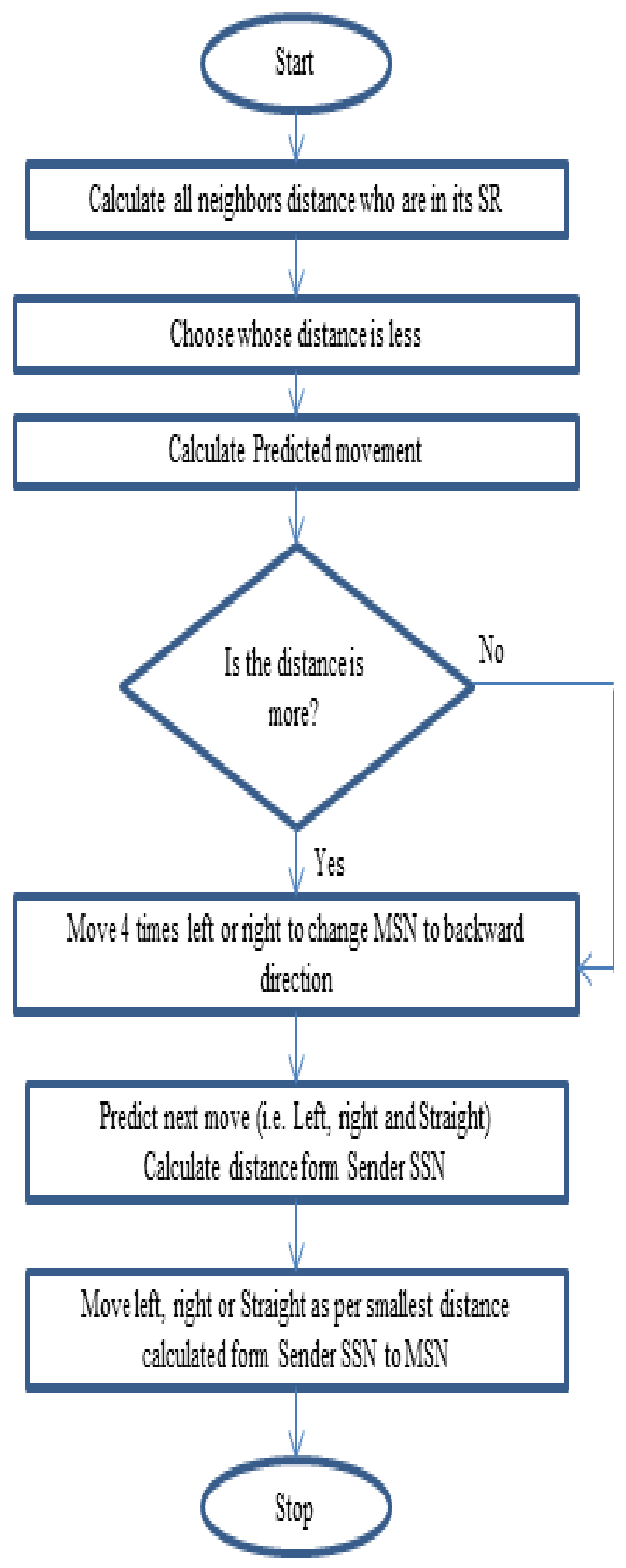

Figure 6. Flowchart of follower's algorithm.

\section{Results and Discussions}

Matlab 7.0 simulator is used for the experiment. Due to the random movement of MSNs and T, the number of iterations/steps cannot be predicted. A number of times simulation is executed and tested for finding the T. The assumed parameters with respective values for the simulation are given in Table 2 .

Two Scenarios are considered for the simulations.

Scenario 1: Simulation having 5 SSNs and 2 MSNs, and

Scenario 2: Simulation having 9 SSNs and 3 MSNs.

While moving, MSNs maintain at least 2SR distance from each other and will be in the CR of other MSN. T is placed randomly in searching space. The speed of $\mathrm{T}$ is same as speed MSNs. If the T is in the SR of any MSN then it is assumed that $\mathrm{T}$ is found. The total energy required for finding the target is calculated by simulation Target and its movements are shown by red dots. Blue, green and pink dots are showing the path of MSNs.

\subsection{Scenario 1}

SSNs are localized in searching space in such a way that whole area is covered. Searching space is divided into four quadrantss and four SSNs are placed at center of each quadrant (SSN1- $(2.5,2.5), \operatorname{SSN} 2-(2.5,7.5)$, SSN3-(7.5, 2.5) and SSN4-(7.5, 7.5)) and fifth SSN is placed at the centre of searching space (SSN5-(5, 5)). MSNs are localized at left-lower corner of searching space (MSN1-(0.3, $0.9)$ and MSN2- $(0.3,0.3))$. Figure $7(a)$ shows the actual localization of SSNs, MSNs, and T in scenario-1.

\subsection{Scenario 2}

Total 9 SSNs (S1 to S9) are localized in searching space in such a way that entire area is covered. Three MSNs (M1 to M3) are localized at left-lower corner of searching space (M1-(0.3, 0.9) and M2- $(0.3,0.3)$ and M3- $(0.9,0.3)$ ). Figure 7(b) shows the actual localization of SSNs, MSNs, and $\mathrm{T}$ in scenario 2 .

In the simulation, the movement of target is shown in red coloured dots, movement of MSN1 is shown in blue coloured dots and movement of MSN2 is shown in green 
Table 2. Parameters and values

\begin{tabular}{|c|c|c|}
\hline Sl. No. & Parameter & Value \\
\hline 1. & Searching Space: & $10 \times 10$ (Obstacle free) \\
\hline 2. & Total Number of MSNs: & 2 \\
\hline 3. & Total Number of SSNs: & 5 \\
\hline 4. & Total Number of Target (T): & 1 \\
\hline 5. & Location of T: & Unknown \\
\hline 6. & Communication Range (CR) of sensors: & 1.2 \\
\hline 7. & Searching Range (SR) of sensors: & 0.3 \\
\hline 8. & Targets Initial Position: & Random \\
\hline 9. & MSNs and Target Movement: & Random \\
\hline 10. & The speed of Target: & Same as MSN \\
\hline 11. & GPS enabled Sensors: & Yes \\
\hline 12. & Maximum Iterations: & 1000 \\
\hline
\end{tabular}
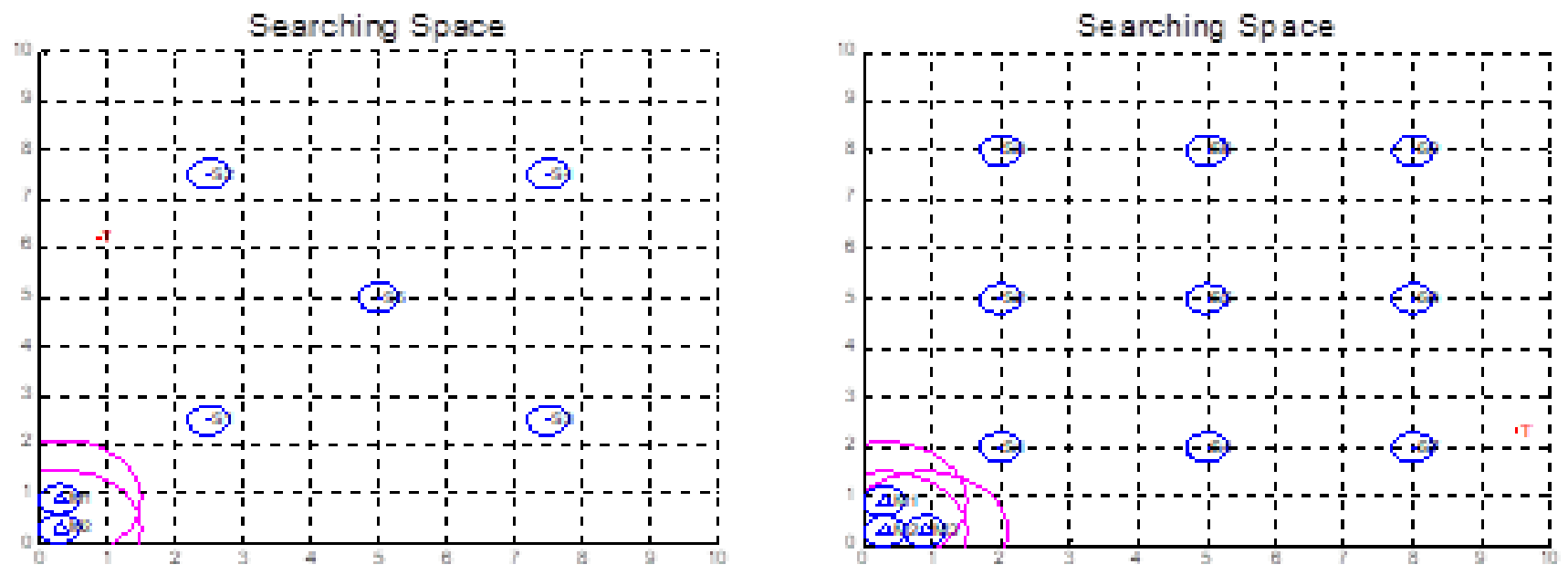

Figure 7. Localization of SSNs, MSNs, and target in simulation.

coloured dots. Blue circles and pink circles indicate the SR and CR of respective sensor. In SSN, CR is not visible for the sake of visibility of MSN's and T's movement but it is same as CR of SSN.
Simulations readings are observed after $\mathrm{T}$ comes in SR of any SSN or MSN. It is assumed that if T moves in SR of any MSN, then searching mission gets completed and it shows total number of iterations required to find the $\mathrm{T}$. 
A number of simulations are carried out of each scenarioand some of the cases are discussed here.

\subsection{Simulations of Scenario 1}

Figure 8(a) shows the initial positions of MSNs, SSNs ad $\mathrm{T}$, after 1 iteration T reaches into the SR of SSN 4 is shown in Figure 8(b). Immediately SSN 4 broadcasts its location and message 'T found' to all MSNs. After receiving the message of SSN, MSNs stop searching and switch into the Leader-follower mode and reach to the sender SSN. After reaching to SSN, again MSNs start searching using PSO technique. Figure 8 (c) shows that $\mathrm{T}$ is found by MSNs after 228 iterations. Figure 9-10 shows another two simulations to find the $\mathrm{T}$.

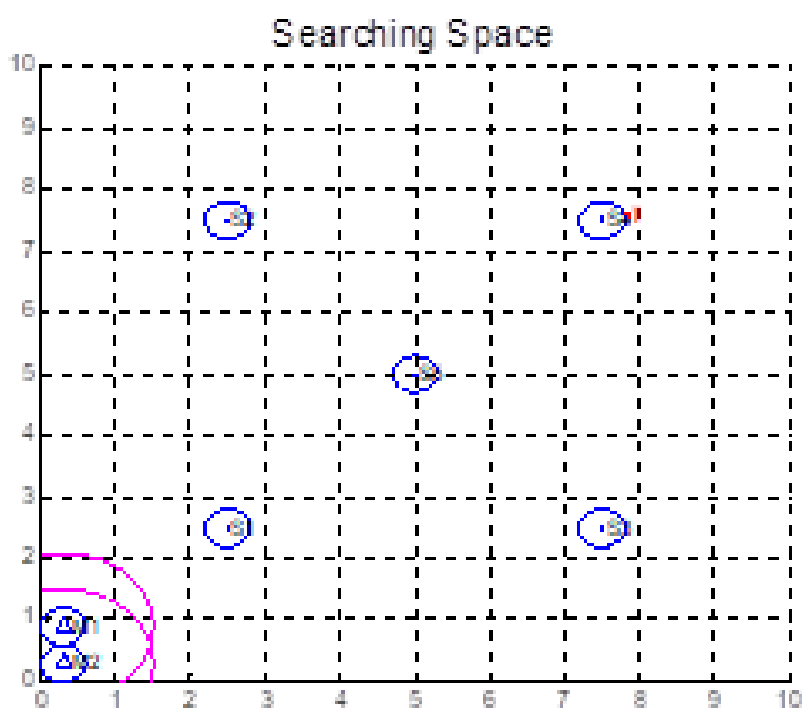

(a)

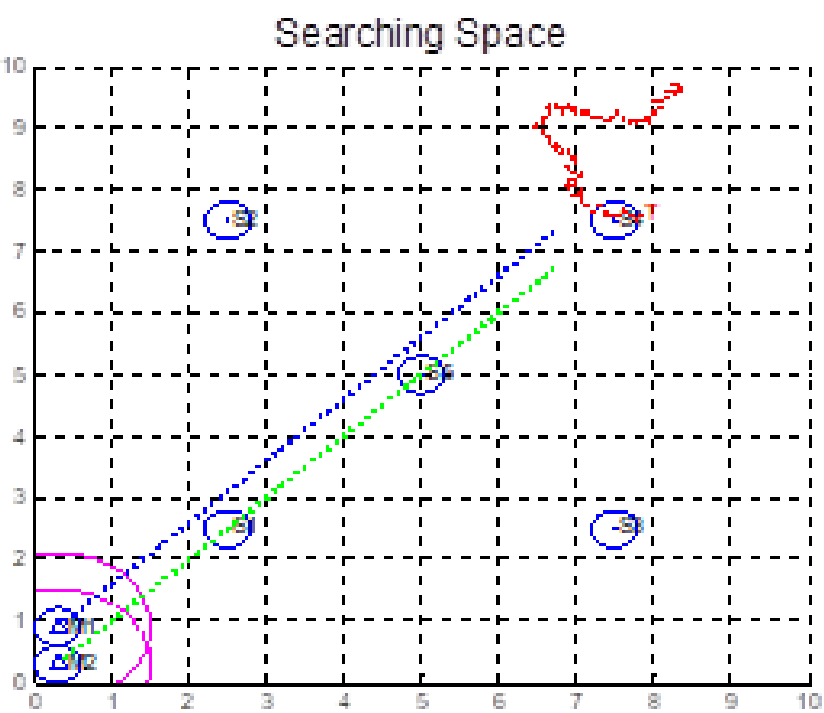

(b)

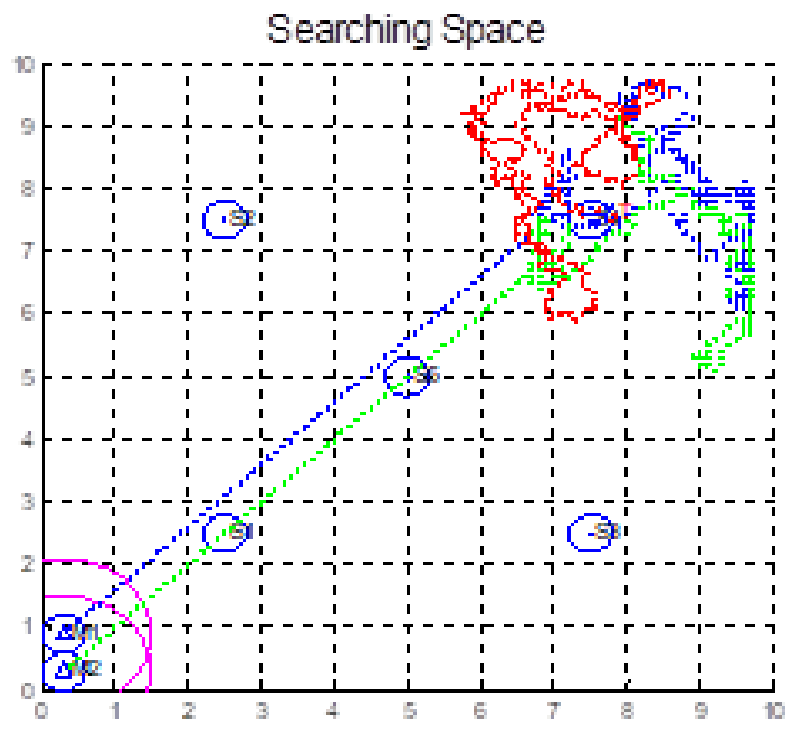

(c)

Figure 8. Case 1: Simulation of scenario1. a) Initial Position (b) T found by SSN 4 after 1 iterations (c) T found by MSN after 228 iterations 


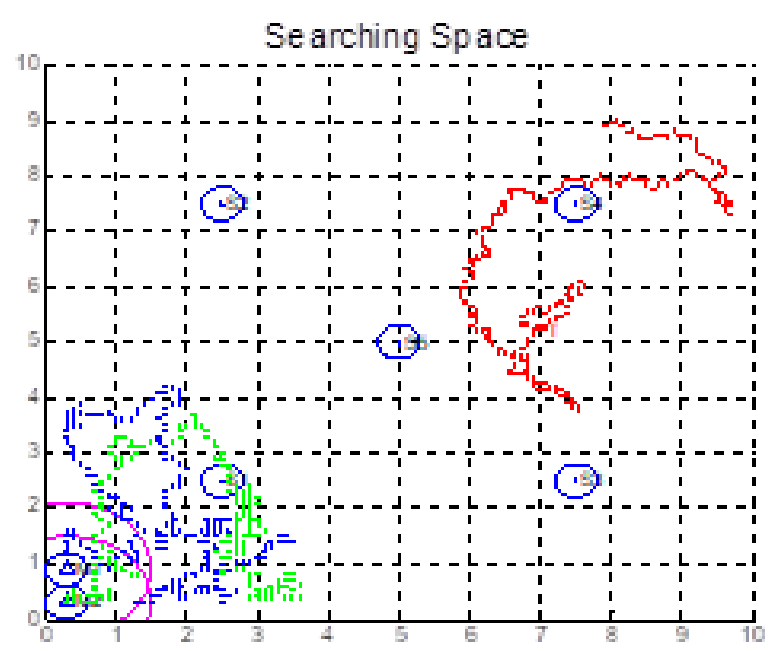

(a)

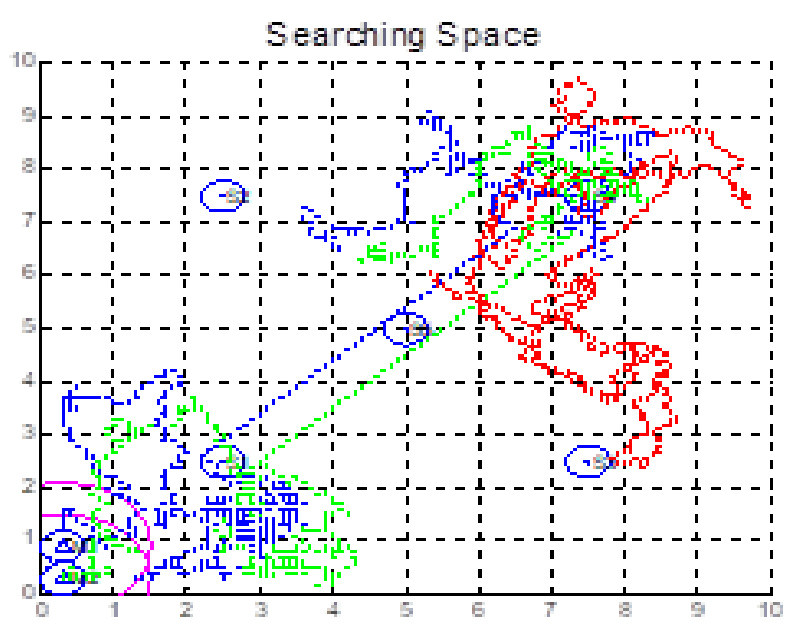

(b)

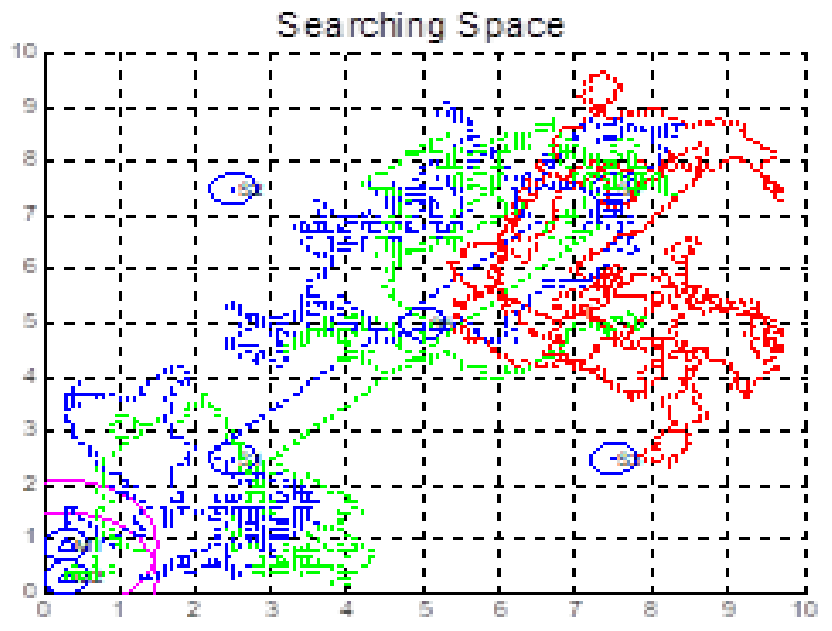

(c)

Figure 9. Case 2: Simulation of scenario 1. (a) Initial Position (b) after 500 iterations c) T found by MSN after 772 iterations

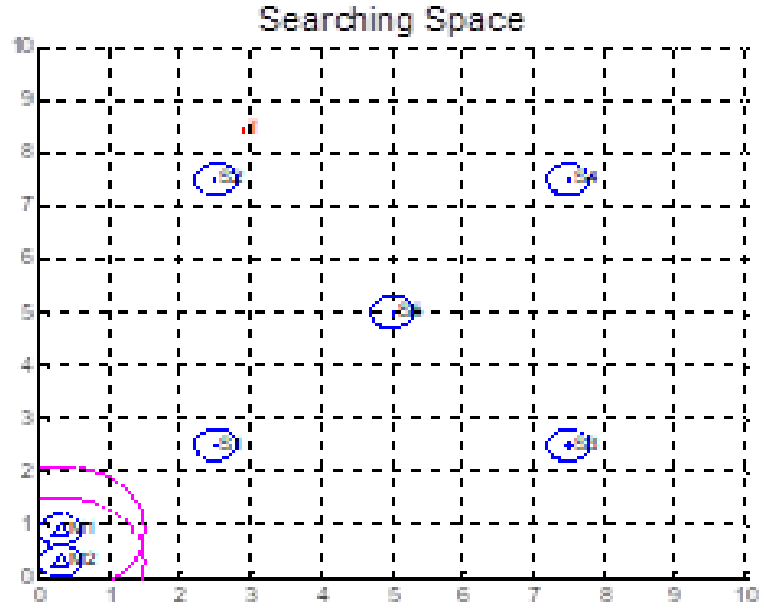

(a)

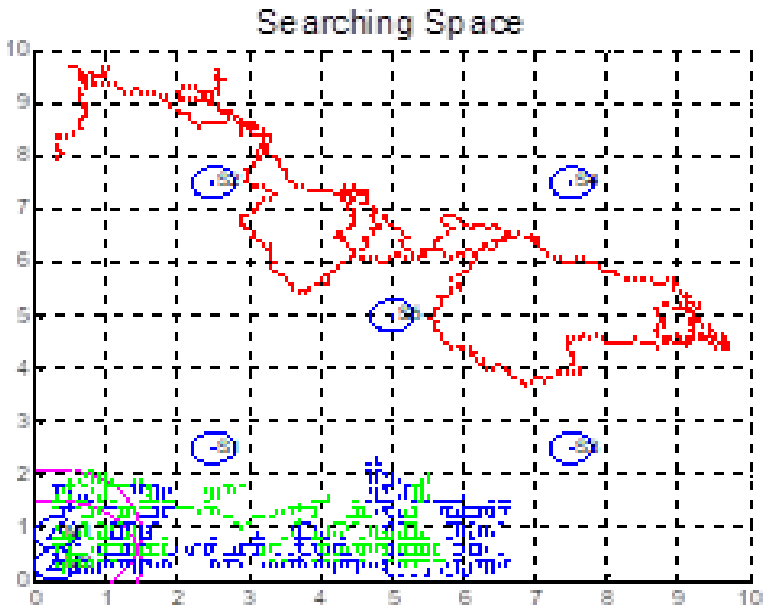

(b) 


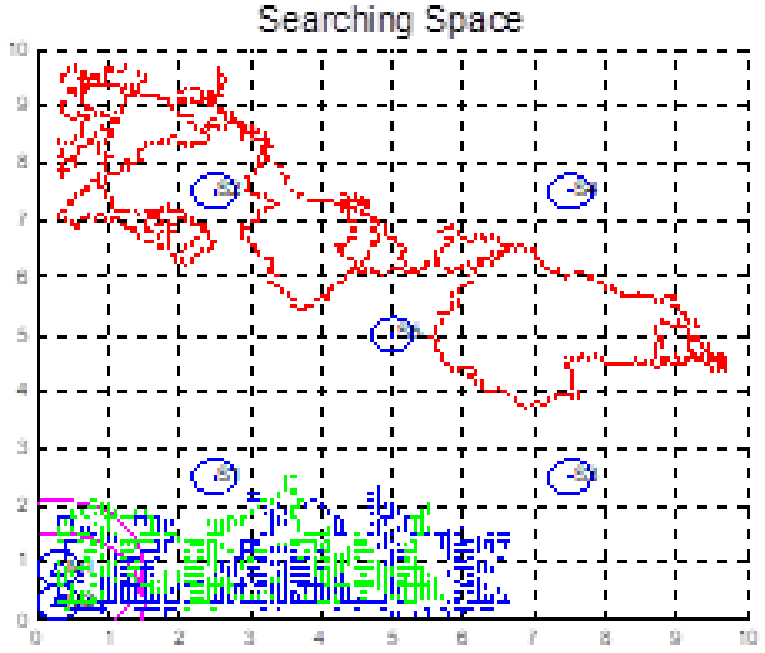

(c)

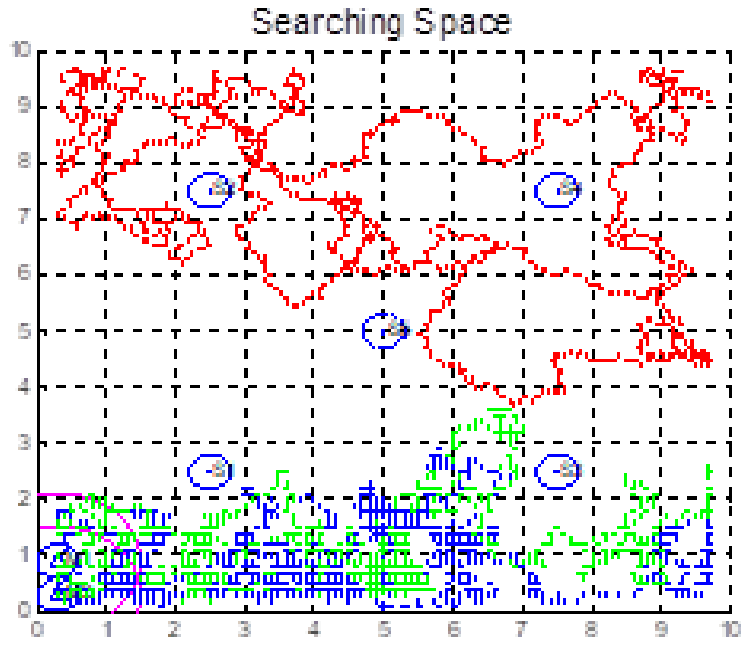

(d)

Figure 10. Case 3: Simulation of scenario 1. (a) Initial positions (b) after 500 iterations (c) After 700 iterations (d) After 100 iterations (Target not found)

\subsection{Simulations of Scenario 2}

Figure 11(a) shows the initial localization of MSNs, SSNs ad T. Figure 11(b) shows that after 2 iterationss, T comes into the range of SSN7. Once T is in the SR of SSN7, it broadcasts its location and message 'T found' to all MSNs. After receiving the message of SSN, MSNs stop searching and switch into the Leader-follower mode and reach to the sender SSN. After reaching to SSN, again MSNs start

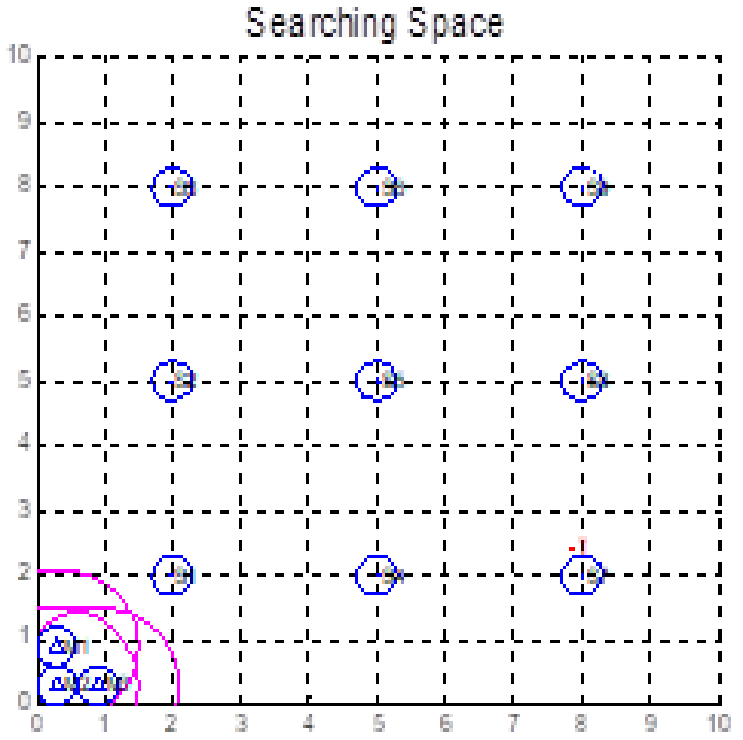

(a)

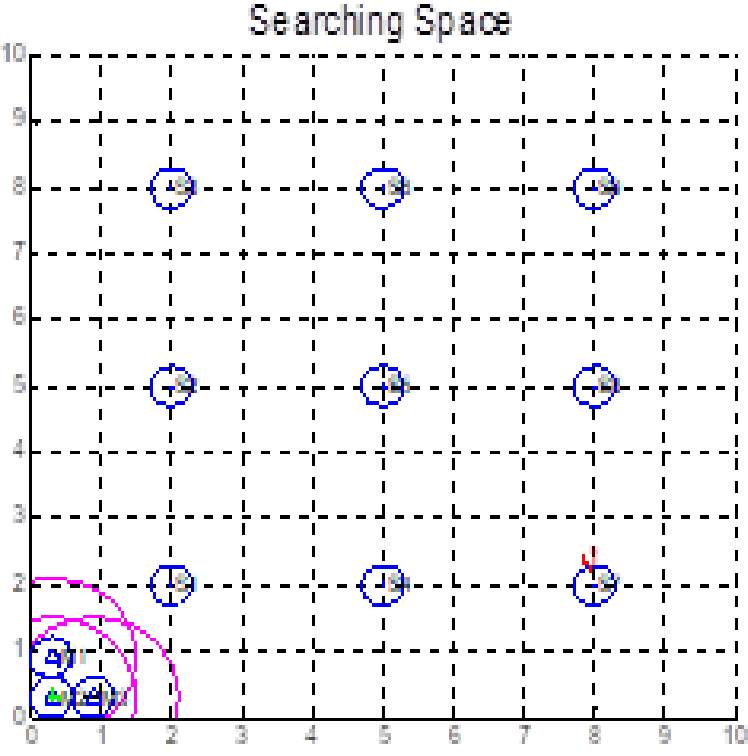

(b) 


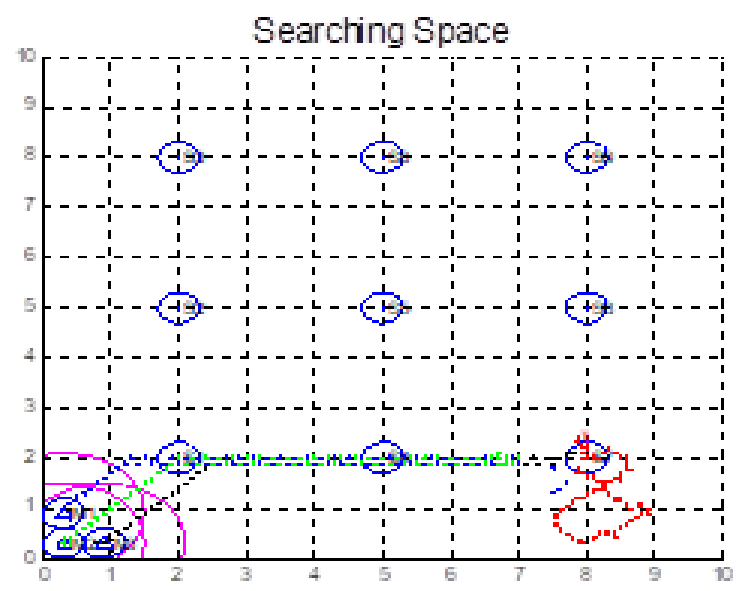

(c)

Figure 11. Case 1: Simulation of scenario 2. (a) Initial Positions (b)T found by SSN 7 after 2 iterations c) T found by MSN after 45 iterations

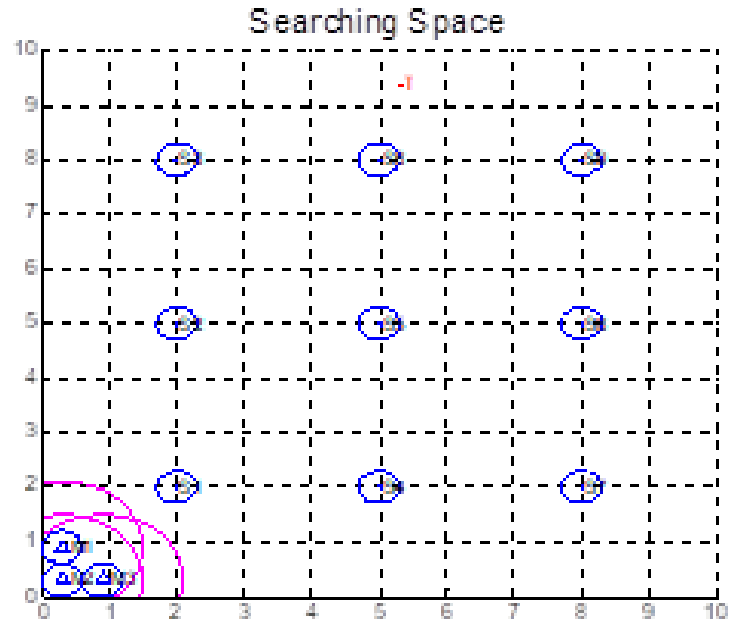

(a)

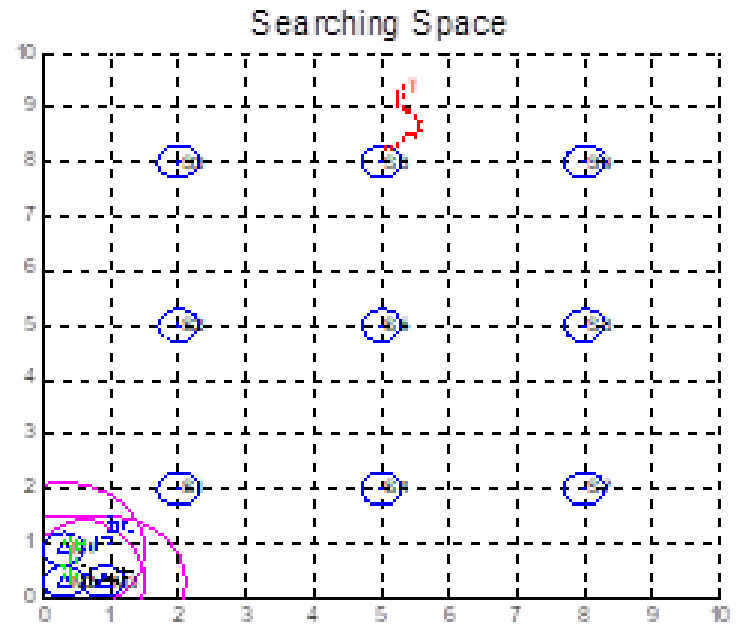

(b)

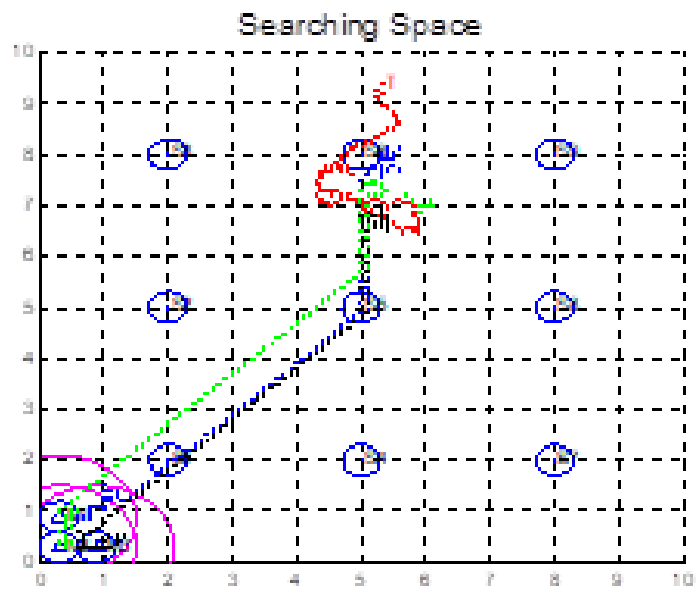

(c)

Figure 12. Case 2: Simulation of scenario 2. (a)Initial Positions (b)T found by SSN 6 after 17 iterations (c) T found by MSN after 59 


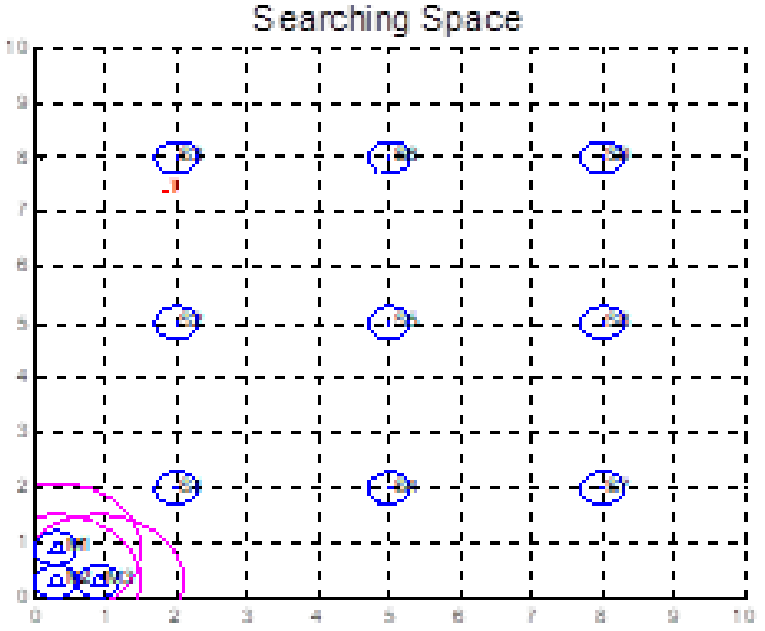

(a)

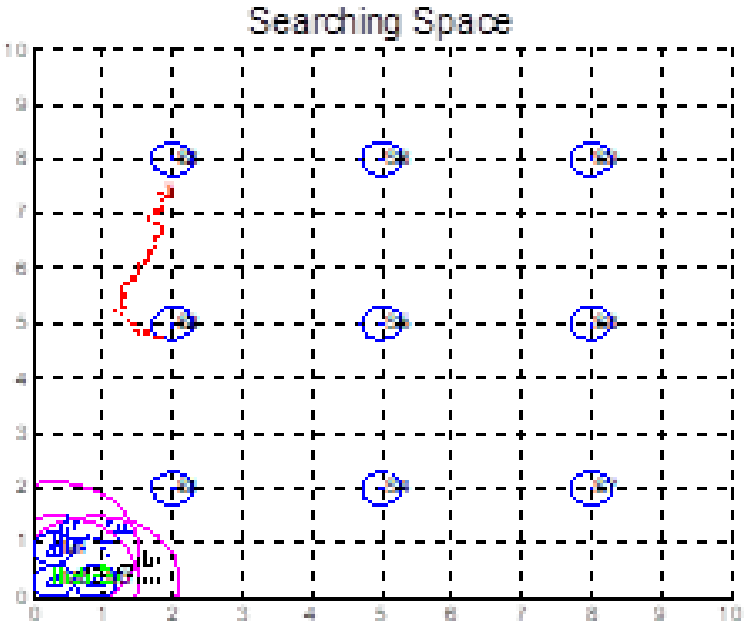

(b)

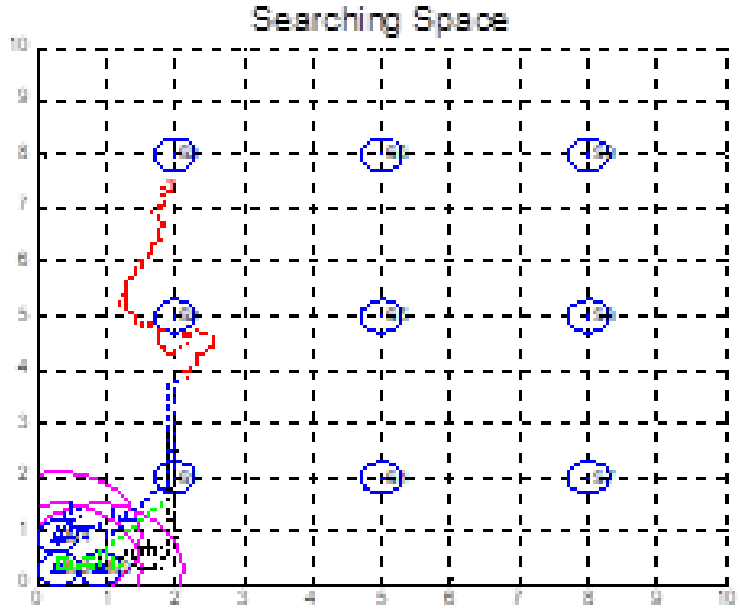

(c)

Figure 13. Case 3: Simulation of Scenario 2. (a) Initial positions (b) T found by SSN 2 after 37 Iterations (c) T found by MSN after 63

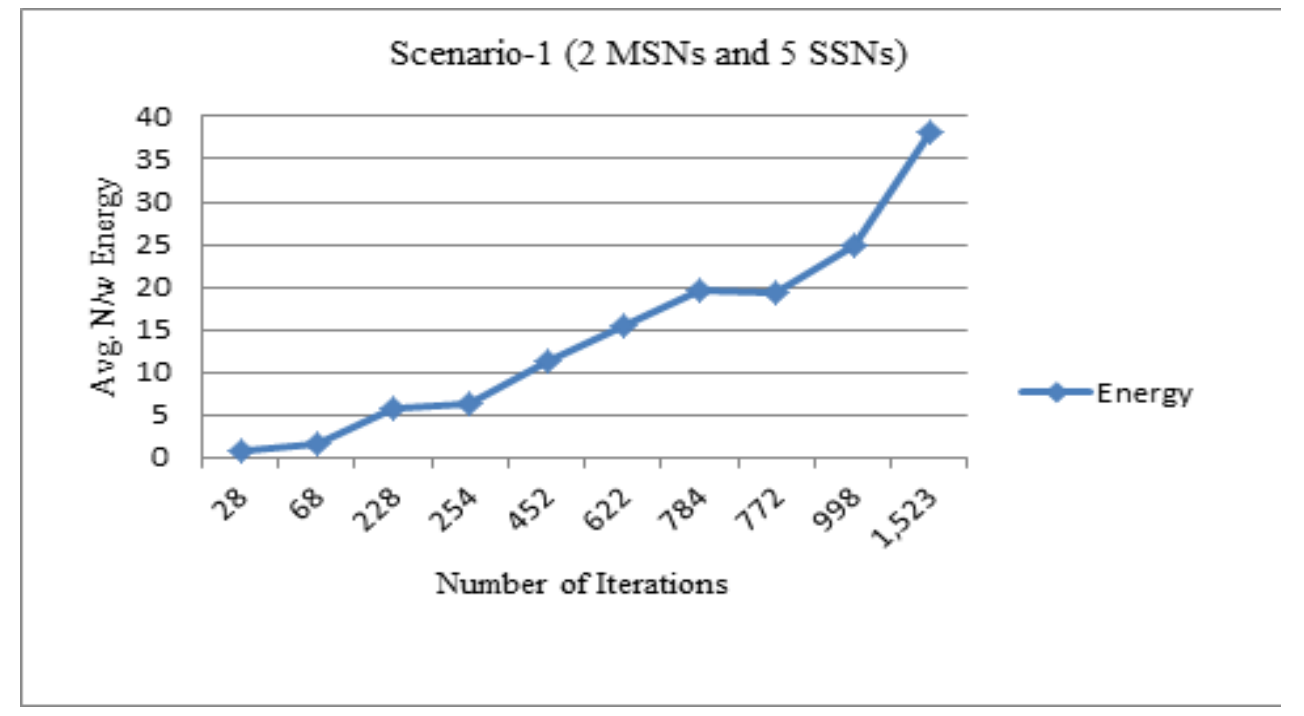

Figure 14. Average network energy of scenario 1. 


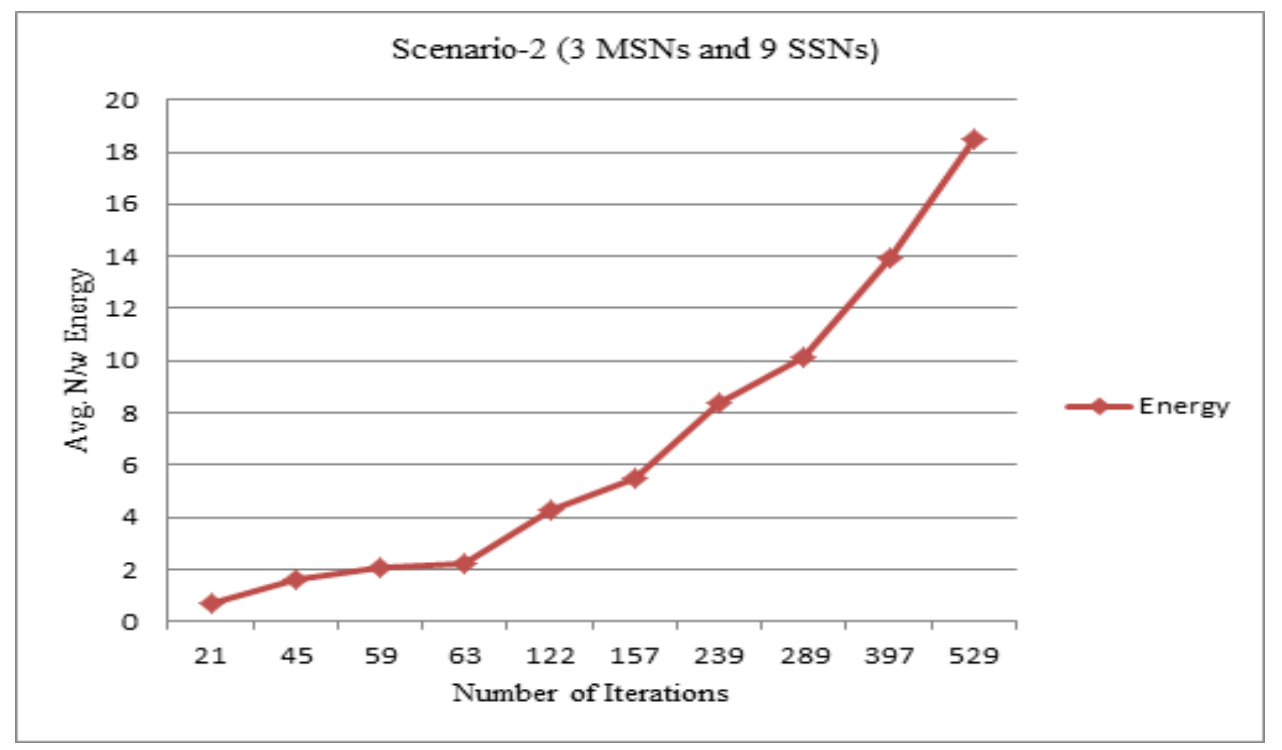

Figure 15. Average network energy of scenario 2.

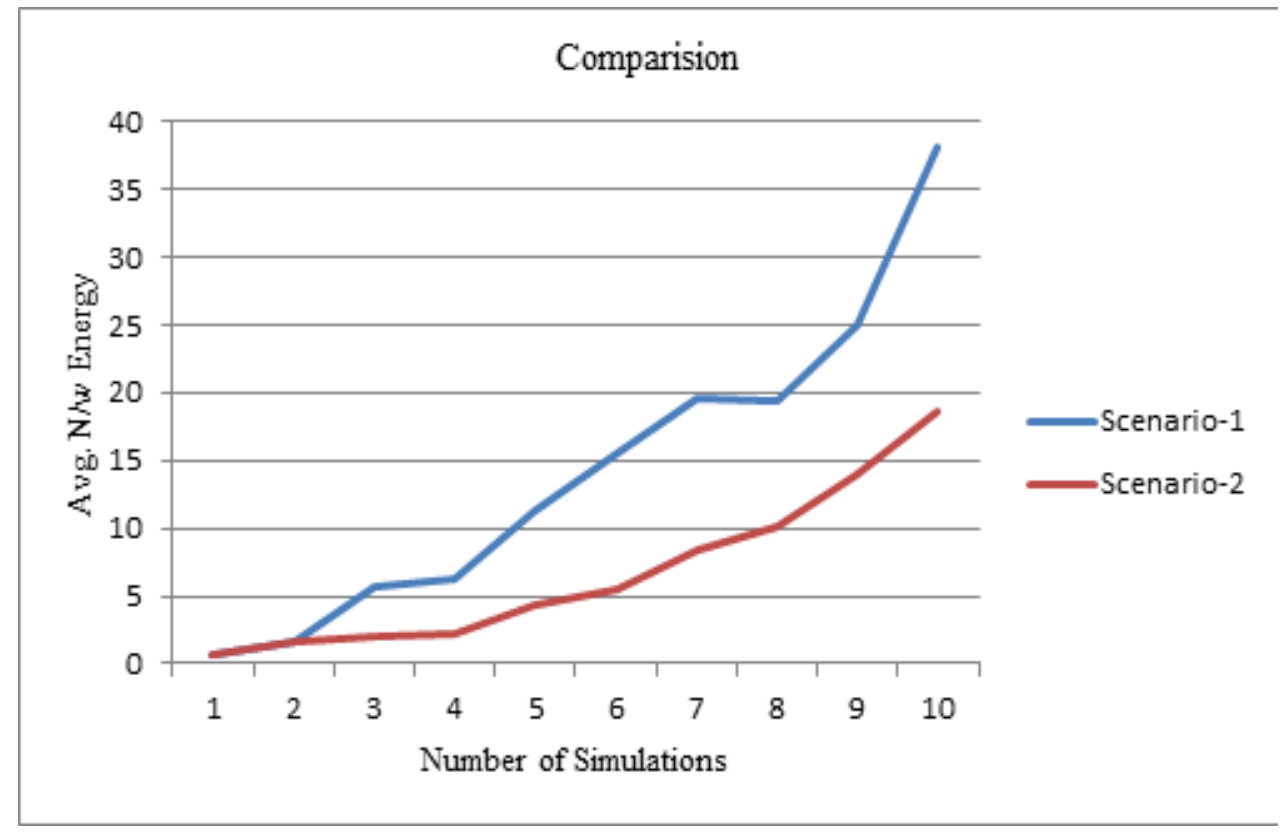

Figure 16. Comparisionof two scenarios in terms of average network energy.

searching using PSO technique. Figure 11(c) shows that $\mathrm{T}$ is found by MSNs after 45 iterations. In same fashion Figure 12-13 shows other two simulations.

After executing too many simulations of both scenarios, some simulation's number of iterations and required average energy is shown in Table 3. In the first scenario which has 2 MSNs and 5 SSNs require more number of iterations so ultimately more energy is required to find the T. But if number of MSNs and SSNs are increased, $\mathrm{T}$ can be tracked with less time and less energy will be drained. It is shown in Figure 14-16. 
Table 3. Average energy of simulations

\begin{tabular}{|c|c|c|c|c|}
\hline \multirow[b]{2}{*}{ Sl. No. } & \multicolumn{2}{|c|}{ Scenario 1} & \multicolumn{2}{|c|}{ Scenario 2} \\
\hline & $\begin{array}{l}2 \text { MSNs and } 5 \\
\text { SSNs (Number of } \\
\text { Iterations) }\end{array}$ & $\begin{array}{l}\text { Average Energy of } \\
\text { simulation }\end{array}$ & $\begin{array}{l}3 \text { MSNs and } 9 \\
\text { SSNs (Number of } \\
\text { Iterations) }\end{array}$ & $\begin{array}{l}\text { Average Energy of } \\
\text { simulation }\end{array}$ \\
\hline 1. & 28 & 0.7 & 21 & 0.74 \\
\hline 2. & 68 & 1.7 & 45 & 1.58 \\
\hline 3. & 228 & 5.7 & 59 & 2.07 \\
\hline 4. & 254 & 6.35 & 63 & 2.21 \\
\hline 5. & 452 & 11.3 & 122 & 4.27 \\
\hline 6. & 622 & 15.55 & 157 & 5.50 \\
\hline 7. & 784 & 19.6 & 239 & 8.37 \\
\hline 8. & 772 & 19.3 & 289 & 10.12 \\
\hline 9. & 998 & 24.95 & 397 & 13.90 \\
\hline 10. & 1,523 & 38.075 & 529 & 18.52 \\
\hline
\end{tabular}

\section{Conclusion and Future Scope}

To find the target in an unknown environment, E-BHAS technique gives good results. In the network, SSNs are placed to give only information about target's presence in the searching range of its own. With the help of SSNs, information MSNs finds the target easily. Even though the the target is not traveling to a SR of any SSN, MSN finds the target by PSO technique. Two scenarios are considered to prove the work. First has only 2 MSNs and 5 SSNs and second has 3 MSNs and 9 SSNs. Results show that if more number of SSNs are planted in surveillance area then the the time of searching and energy reduces.

In future some obstacles are planned to put in between the the path of MSN and target and some noise will be considered while moving MSNs. Target speed is assumed same as the speed of MSNs so it is planned to vary the the speed of the the target. Also in future multiple targets are planned to use for simulation.

\section{References}

1. Wang X, Wang S. Collaborative signal processing for target tracking in distributed wireless sensor networks, Journal of Parallel and Distributed Computing. 2007; 67(5):501-15. Crossref.

2. Wang X, Wang S, Ma J. An improved co-evolutionary particle swarm optimization for wireless sensor networks with dynamic deployment, Sensors. 2007; 7(3):354-70. Crossref.

3. Ferrante E, Turgut AE, Stranieri A, Pinciroli C, Birattari M, Dorigo M. A self-adaptive communication strategy for flocking in stationary and non-stationary environments, Natural Computing. 2014; 13(2):225-45. Crossref.

4. Ren H, Meng MMQH. Biologically Inspired Approaches for Wireless Sensor Networks. Proceeding IEEE International Conference, Mechatronics and Automation; 2006. p. 76268. Crossref.

5. Kennedy J, Eberhart RC. Particle swarm optimization, Proceeding IEEE Conference on Neural Networks. 1995. 11:1942-48. Crossref.

6. Eberhart RC, Kennedy J. A new optimizer using particle swarm theory. Proceedings on international symposium on 
micro machine and human science, IEEE Service Center, USA; 1995. p. 39-43. Crossref.

7. Kennedy J. The particle swarm: social adaptation of knowledge. Proceedings of IEEE international conference on evolutionary computation USA; 1997. p. 303-08. Crossref.

8. Anderson C, Franks NR. Teams in animal society's behavior, Ecology. 2001; 12(5):534-40.

9. Lissaman PBS, Shollenberger CA. Formation flight of birds, Science. 1970; 168(3934):1003-05. Crossref.

10. Tseng PH, Feng KT, Lin YC, Chen CL. Wireless location tracking algorithms for environments with insufficient signal sources, IEEE Transaction Mobile Computing. 2009; 8(12):1676-89. Crossref.

11. Li T, Ekpenyong A, Huang YF. Source localization and tracking using distributed asynchronous sensors, IEEE Transaction Signal Processing. 2006; 54(10):3991-4003. Crossref.

12. Yang CD, Yang CC. A Unified approach to proportional navigation, IEEE Trans. Aerospace and Electronic Systems. 1997; 33(2):557-67. Crossref.

13. Mehrandezh M, Sela MN, Fenton RG, Benhabib B. Proportional navigation guidance for robotic interception of moving objects, Journal of Robotic Systems. 2000; 17(6):321-40. Crossref.

14 Zou Y, Chakrabarty K. Distributed mobility management for target tracking in mobile sensor networks, IEEE Trans. Mobile Computing. 2007; 6(8):872-87. Crossref.

15. Fu Y, Ling Q, Tian Z. Distributed sensor allocation for multi-target tracking in wireless sensor networks, IEEE Transactions on Aerospace and Electronic Systems. 2012; 48(4):3538 - 53. Crossref.

16. Cai Y, Yang SX, Xu X. A combined Hierarchical Reinforcement Learning Based Approach for Multi-Robot Cooperative Target Searching in Complex Unknown Environments. IEEE Symposium on Adaptive Dynamic Programming and Reinforcement Learning (ADPRL); 2013. p. 52-59. Crossref. PMCid: PMC4150634.

17. Cai Y, Yang SX, Xu X, Mittal GS. A Hierarchical Reinforcement Learning Based Approach for Multi-robot Cooperation in Unknown Environments. Proceedings of the 2011 2nd International Congress on Computer Applications and Computational Science; 2011. P. 69-74.

18. Xu E, Ding Z, Dasgupta S. Target tracking and mobile sensor navigation in wireless sensor networks, IEEE Transactions on Mobile Computing. 2013; 12(1):177-86. Crossref.

19. Liu B, Dousse O, Nain P, Towsley D. Dynamic coverage of mobile sensor networks, IEEE Transaction Parallel Distribution System. 2013; 24(2):301-11. Crossref.

20. Tan R, Xing G, Wang J, So C. Exploiting reactive mobility for collaborative target detection in wireless sensor networks, IEEE Transaction Mobile Computing. 2010; 9(3):317-32. Crossref.

21. Fu Z, You K. Optimal mobile sensor scheduling for a guaranteed coverage ratio in hybrid wireless sensor networks, International Journal of Distributed Senser Network. 23Apr 2013; 11. Article ID 740841. Crossref. .

22. Zhou S, Wu MY, Shu W. Finding optimal placements for mobile sensors: Wireless sensor network topology adjustment, Proceeding IEEE 6th Circuits System Symposium Emerging Technologies Frontiers Mobile Wireless Communication. 2004; 2:529-32. Crossref.

23. Wang X, Ma JJ, Wang S, Bi DW. Distributed particle swarm optimization and simulated annealing for energy-efficient coverage in wireless sensor networks, Sensors (Basel). 2007; 7(5):628-48. Crossref.

24. Mahboubi SH, Masoudimansour W, Aghdam AG, Sayrafian-Pour K. Maximum lifetime strategy for target monitoring with controlled node mobility in sensor networks with obstacles, IEEE Transactions on Automatic Control. 2016; 61(110):3493-508.

25. Mahboubi H, Masoudimansour W, Aghdam AG, SayrafianPour K. Cost-Efficient Routing with Controlled Node Mobility in Sensor Networks. Proceedings of the IEEE Multi-Conference on Systems and Control; 2011. p. 123843. Crossref.

26. Heinzelman WR, Chandrakasan A, Balakrishnan H. An application specific protocol architecture for wireless micro-sensor networks, IEEE Transactions on Wireless Communications. 2002; 1(4):660-70. Crossref. 